\title{
Decomposition of Optical MIMO Systems using Polynomial Matrix Factorization
}

\author{
A. Ahrens*, A. Sandmann*, S. Lochmann*, Z. Wang ${ }^{\dagger}$ \\ * Hochschule Wismar, University of Applied Sciences - Technology, Business and Design, Philipp-Müller-Straße 14, 23966 \\ Wismar,Germany,E-mail: andreas.ahrens@hs-wismar.de,steffen.lochmann@hs-wismar.de \\ $\dagger$ School of Engineering, Cardiff University, Queen's Buildings, The Parade, Cardiff CF24 3AA, Wales, UK, \\ E-mail: WangZ49@cardiff.ac.uk
}

\begin{abstract}
Keywords: Multiple-Input Multiple-Output (MIMO) Transmission, Optical Fiber Transmission, Multimode Fiber (MMF), Polynomial Matrix Decomposition.
\end{abstract}

\begin{abstract}
Within the last years the multiple-input multiple-output (MIMO) technology has revolutionized the optical fiber community. Theoretically, the concept of MIMO is well understood and shows some similarities to wireless MIMO systems. The interference in broadband MIMO systems can be removed by applying a spatio-temporal vector coding (STVC) channel description and using singular value decomposition (SVD) in combination with signal pre- and post-processing. In this contribution a newly developed SVD algorithm for polynomial matrices (PMSVD) is analyzed and compared to the commonly used SVD-based STVC. The PMSVD is implemented by an iterative polynomial matrix eigenvalue decomposition (PEVD) algorithm, namely the second order sequential best rotation algorithm (SBR2). The bit-error rate (BER) performance is evaluated and optimized by applying bit and power allocation schemes. For our simulations, the specific impulse responses of the $(2 \times 2)$ MIMO channel, including a $1.4 \mathrm{~km}$ multi-mode fiber and optical couplers at both ends, are measured for the operating wavelength of $1576 \mathrm{~nm}$. The computer simulation results show that the PMSVD could be an alternative signal processing approach compared to conventional SVD-based MIMO approaches in frequency-selective MIMO channels.
\end{abstract}

\section{Introduction}

Aiming at further increasing the fiber capacity in optical transmission systems, the concept of MIMO, well studied and wide-spread in radio transmission systems, has led to increased research activities [1,2]. Theoretical investigations have shown that similar capacity increases are possible compared to wireless systems. The basis for this approach is the exploitation of different optical mode groups. MIMO systems have become the subject of extensive research as MIMO is able to support higher data rates and shows a higher reliability than single-input single-output (SISO) systems known from wireless MIMO systems. Singular-value decomposition (SVD) is well-established in MIMO signal processing where the whole MIMO channel is transferred into a number of weighted SISO channels. The unequal weighting of the SISO channels has led to intensive research on bit- and power allocation techniques [3]. The PMSVD is a signal processing technique, decomposing the MIMO channel into a number of independent frequency-selective SISO channels so called layers [4]. The remaining layer-specific interferences as a result of the PMSVD-based signal processing can be easily removed by further signal processing such as zeroforcing equalization or maximum likelihood sequence estimation. The novelty of our contribution is that we demonstrate the benefits of amalgamating a suitable choice of MIMO layers activation and number of bits per layer along with the appropriate allocation of the transmit power under the constraint of a given fixed data throughput.

The remaining parts of this paper are structured as follows: Section 2 introduces the state of the art SVD-based MIMO system model. The PMSVD is analyzed in section 3. In section 4 the quality criteria and the proposed power allocation solutions are discussed. The associated performance results are presented and interpreted in section 5 . Finally, section 6 provides some concluding remarks.

\section{State of the Art}

A frequency-selective MIMO link, composed of $n_{\mathrm{T}}$ optical inputs and $n_{\mathrm{R}}$ optical outputs is given by

$$
\boldsymbol{u}=\boldsymbol{H} \cdot \boldsymbol{c}+\boldsymbol{n} .
$$

In equation (1), $\boldsymbol{c}$ is the $\left(N_{\mathrm{T}} \times 1\right)$ transmitted data signal vector containing the complex input symbols transmitted over $n_{\mathrm{T}}$ optical inputs in $K$ consecutive time slots, i. e., $N_{\mathrm{T}}=K n_{\mathrm{T}}$. The vector $\boldsymbol{u}$ describes the $\left(N_{\mathrm{R}} \times 1\right)$ received signal vector, of the length $N_{\mathrm{R}}=\left(K+L_{\mathrm{c}}\right) n_{\mathrm{R}}$, which is extended if compared to the transmitted signal vector based on the $\left(L_{\mathrm{c}}+1\right)$ nonzero elements of the resulting symbol rate sampled overall channel impulse response between the $\mu$ th optical input and $v$ th optical output. The $\left(N_{\mathrm{R}} \times 1\right)$ vector $\boldsymbol{n}$ in (1) describes the noise term [3] and the $\left(N_{\mathrm{R}} \times N_{\mathrm{T}}\right)$ system matrix $\boldsymbol{H}$ of the block-oriented system model results in

$$
\boldsymbol{H}=\left[\begin{array}{ccc}
\boldsymbol{H}_{11} & \cdots & \boldsymbol{H}_{1 n_{\mathrm{T}}} \\
\vdots & \ddots & \vdots \\
\boldsymbol{H}_{n_{\mathrm{R}} 1} & \cdots & \boldsymbol{H}_{n_{\mathrm{R}} n_{\mathrm{T}}}
\end{array}\right]
$$


and consists of $n_{\mathrm{R}} n_{\mathrm{T}}$ SISO channel matrices $\boldsymbol{H}_{v \mu}$ (with $v=1, \ldots, n_{\mathrm{R}}$ and $\left.\mu=1, \ldots, n_{\mathrm{T}}\right)$ [5]. Each of these matrices $\boldsymbol{H}_{v \mu}$ with the dimension $\left(\left(K+L_{\mathrm{c}}\right) \times K\right)$ describes the influence of the channel from the $\mu$ th transmitter to the $v$ th receiver including transmit and receive filtering.

By using SVD, the system matrix $\boldsymbol{H}$ can be written as $\boldsymbol{H}=\boldsymbol{S} \cdot \boldsymbol{V} \cdot \boldsymbol{D}^{\mathrm{H}}$, where $\boldsymbol{S}$ and $\boldsymbol{D}^{\mathrm{H}}$ are unitary matrices and $\boldsymbol{V}$ is a real-valued diagonal matrix of the positive square roots of the eigenvalues of the matrix $\boldsymbol{H}^{\mathrm{H}} \boldsymbol{H}$ sorted in descending order. The conjugate transpose (Hermitian) of $\boldsymbol{D}$ is denoted by $\boldsymbol{D}^{\mathrm{H}}$. In order to remove the interferences, the MIMO data vector $\boldsymbol{c}$ is now multiplied by the matrix $\boldsymbol{D}$ before transmission. In turn, the receiver multiplies the received vector $\boldsymbol{u}$ by the matrix $\boldsymbol{S}^{\mathrm{H}}$. Thereby neither the transmit power nor the noise power is enhanced given $\boldsymbol{S}$ and $\boldsymbol{D}$ are unitary. The overall transmission relationship is defined as

$$
\boldsymbol{y}=\boldsymbol{S}^{\mathrm{H}}(\boldsymbol{H} \cdot \boldsymbol{D} \cdot \boldsymbol{c}+\boldsymbol{n})=\boldsymbol{V} \cdot \boldsymbol{c}+\boldsymbol{w} .
$$

As a consequence of the processing in (3), the channel matrix $\boldsymbol{H}$ is transformed into independent, non-interfering layers having unequal gains.

With the proposed system structures, the SVD-based equalization leads to a different number of MIMO layers $\ell$ (with $\ell=1,2, \ldots, L$ ) at the time $k$ (with $k=1,2, \ldots, K$ ) as shown in Figure 1.

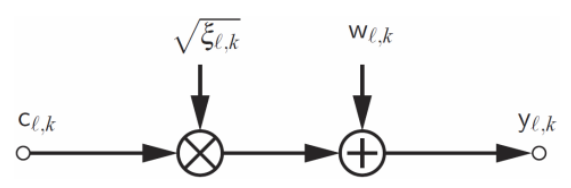

Figure 1. Resulting layer-specific SVD-based broadband MIMO system model

Here it is worth noting that the number of parallel transmission layers $L$ at the time-slot $k$ is limited by $\min \left(n_{\mathrm{T}}, n_{\mathrm{R}}\right)$. The complex-value data symbol $c_{\ell, k}$ to be transmitted over the layer $\ell$ at the time $k$ is now weighted by the corresponding positive real-valued singular-value $\sqrt{\xi_{\ell, k}}$ and further disturbed by the additive white Gaussian noise term $w_{\ell, k}$.

\section{Polynomial Matrix Factorization}

In contrast to the STVC, the polynomial matrix factorization exploits the description of the channel impulse responses in the z-domain. Thus, each frequency selective channel impulse response $h_{v \mu}(k)$ between the $\mu$ th optical input and the $v$ th optical output of a $\left(n_{\mathrm{R}} \times n_{\mathrm{T}}\right)$ MIMO system is given by

$$
\underline{h}_{v \mu}(z)=\sum_{k=0}^{L_{\mathrm{c}}} h_{v \mu}[k] z^{-k}
$$

where the underscore denotes a polynomial. Grouping these impulse responses into the polynomial channel matrix forms the broadband MIMO channel and thus it can be described as multiple non-polynomial matrices $\boldsymbol{H}_{k}$ multiplied with their respective delay $z^{-k}$ as follows

$$
\underline{\boldsymbol{H}}(z)=\sum_{k=0}^{L_{\mathrm{c}}} \boldsymbol{H}_{k} z^{-k}=\left[\begin{array}{ccc}
\underline{h}_{11}(z) & \cdots & \underline{h}_{1 n_{\mathrm{T}}}(z) \\
\vdots & \ddots & \vdots \\
\underline{h}_{n_{\mathrm{R}} 1}(z) & \cdots & \underline{h}_{n_{\mathrm{R}} n_{\mathrm{T}}}(z)
\end{array}\right],
$$

where $\underline{\boldsymbol{H}}(z) \in \underline{\mathbb{C}}^{n_{\mathrm{R}} \times n_{\mathrm{T}}}$. Using this polynomial description in the $\mathrm{z}$-domain, a MIMO system is described in analogy to (1) by

$$
\underline{\boldsymbol{u}}(z)=\underline{\boldsymbol{H}}(z) \underline{\boldsymbol{c}}(z)+\underline{\boldsymbol{n}}(z),
$$

where $\underline{\boldsymbol{c}}(z)$ is the transmit signal vector, $\underline{\boldsymbol{u}}(z)$ is the receive signal vector and $\underline{\boldsymbol{n}}(z)$ describes the additive white Gaussian noise (AWGN) component in polynomial notation.

By calculating the PMSVD the polynomial channel matrix $\underline{\boldsymbol{H}}(z)$ can be orthogonalized. Here, an iterative polynomial matrix eigenvalue decomposition algorithm is used, namely the second-order sequential best rotation (SBR2) algorithm [4]. The decomposition of the polynomial channel matrix results in $\underline{\boldsymbol{H}}(z)=\underline{\boldsymbol{S}}(z) \underline{\boldsymbol{V}}(z) \underline{\widetilde{\boldsymbol{D}}}(z)$, where $(\tau)$ denotes the para-conjugate operator. The matrices $\underline{\boldsymbol{S}}(z) \in \underline{\mathbb{C}}^{n_{\mathrm{R}} \times n_{\mathrm{R}}}$ and $\underline{\widetilde{D}}(z) \in \underline{\mathbb{C}}^{n_{\mathrm{T}} \times n_{\mathrm{T}}}$ are para-unitary matrices and $\underline{\boldsymbol{V}}(\bar{z}) \in \underline{\mathbb{C}}^{n_{\mathrm{R}} \times n_{\mathrm{T}}}$ is ideally a diagonal matrix of the form (for $n_{\mathrm{T}}=n_{\mathrm{R}}$ )

$$
\underline{\boldsymbol{V}}(z)=\left[\begin{array}{cccc}
\underline{v}_{1}(z) & 0 & \cdots & 0 \\
0 & \underline{v}_{2}(z) & \cdots & 0 \\
\vdots & \vdots & \ddots & \vdots \\
0 & 0 & \cdots & \underline{v}_{L}(z)
\end{array}\right],
$$

where the diagonal polynomial elements at the layer $\ell$ are described by $\underline{v}_{\ell}(z)=\sum_{k=0}^{L_{\mathrm{V}}} v_{\ell, k} z^{-k}$. In contrast to the singular values $\sqrt{\xi_{\ell, k}}$ using SVD, the polynomial coefficients of $\underline{v}_{\ell}(z)$ are complex. In analogy to the SVD model, the maximal number of activated layers $L$ using PMSVD is given by $\min \left\{n_{\mathrm{R}}, n_{\mathrm{T}}\right\}$. To eliminate the interferences, signal preprocessing at the transmitter and post-processing at the receiver has to be applied in analogy to the classical SVD. Consequently, the transmit data vector $\underline{\boldsymbol{c}}(z)$ is multiplied by $\underline{\boldsymbol{D}}(z)$ and the receive vector $\underline{\boldsymbol{u}}(z)$ is multiplied by $\underline{\tilde{\boldsymbol{S}}}(z)$ such that

$$
\underline{\boldsymbol{y}}(z)=\underline{\tilde{\boldsymbol{S}}}(z)(\underline{\boldsymbol{H}}(z) \underline{\boldsymbol{D}}(z) \underline{\boldsymbol{c}}(z)+\underline{\boldsymbol{n}}(z)) .
$$

Analog to (3), the orthogonalized system results in [6]

$$
\underline{\boldsymbol{y}}(z)=\underline{\boldsymbol{V}}(z) \underline{\boldsymbol{c}}(z)+\underline{\boldsymbol{w}}(z)
$$

The resulting system is described by multiple SISO channels. The layer based discrete-time description is expressed as

$$
y_{\ell}(k)=v_{\ell}(k) * c_{\ell}(k)+w_{\ell}(k) .
$$

The parameter $L_{\mathrm{v}}+1$ describes the number of non-zero coefficients of the layer-specific impulse responses. The layer-specific model is depicted in Figure 2. 


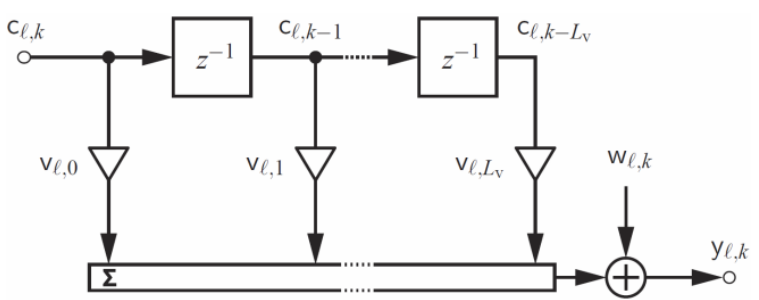

Figure 2. Resulting layer-specific PMSVD-based broadband MIMO system model

Here in each layer the input symbols $c_{\ell}(k)$ are influenced by a finite impulse response filter $v_{\ell}(k)=\left(v_{\ell, 0}, v_{\ell, 1}, \ldots, v_{\ell, L_{\mathrm{V}}}\right)$ and hence inter symbol interference (ISI) occurs on each layer. In order to remove the ISI a corresponding T-spaced zero forcing equalizer $f_{\ell}(k)$ is applied to the received signal as follows

$$
z_{\ell}(k)=y_{\ell}(k) * f_{\ell}(k) .
$$

Accordingly, the equalized receive signal results in

$$
z_{\ell}(k)=c_{\ell}(k)+w_{\ell}(k) * f_{\ell}(k) .
$$

The corresponding layer-specific ISI free system model is shown in Figure 3. The transmitted symbols are received unchanged and the noise $w_{\ell}(k)$ is weighted by the equalizer coefficients $f_{\ell}(k)$. The PMSVD-based broadband MIMO system model with layer-specific T-spaced equalization is entitled as T-PMSVD system model [6].

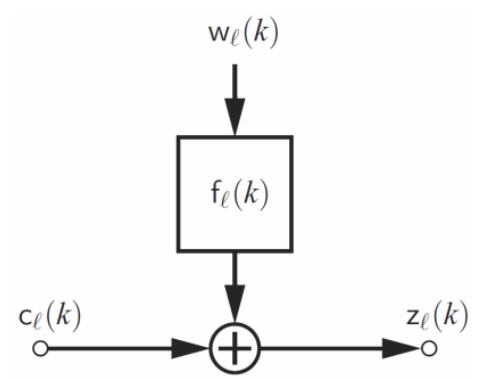

Figure 3. ISI free layer-specific T-PMSVD-based broadband MIMO system model

\section{Resource Allocation}

In general the quality criterion for transmission systems can be expressed with using the signal to noise ratio (SNR) at the detector input as follows

$$
\rho=\frac{(\text { half vertical eye opening })^{2}}{\text { Noise Power }}=\frac{\left(U_{\mathrm{A}}\right)^{2}}{P_{\mathrm{R}}}
$$

where $U_{\mathrm{A}}$ and $P_{\mathrm{R}}$ correspond to one quadrature component. Considering a layer based MIMO system with a given SNR $\rho^{(\ell, k)}$ for each layer $\ell$ and time $k$ the bit-error rate (BER) probability is given by

$$
P_{\mathrm{BER}}^{(\ell, k)}=\frac{2}{\log _{2} M_{\ell}}\left(1-\frac{1}{\sqrt{M_{\ell}}}\right) \operatorname{erfc}\left(\sqrt{\frac{\rho^{(\ell, k)}}{2}}\right) .
$$

This BER is averaged over all time slots and activated layers taking different modulation sizes at each layer into account. When considering time-variant channel conditions, rather than an additive white Gaussian noise (AWGN) channel, the BER can be derived by considering the different transmission block SNRs. For quadrature amplitude modulated signals (QAM) the average transmit power per layer can be expressed as $P_{\mathrm{s}, \ell}=2 / 3 U_{\mathrm{s}, \ell}^{2}\left(M_{\ell}-1\right)$. Intuitively the total available transmit power $P_{\mathrm{S}}$ is equally split between the $L$ activated layers, and hence the layer-specific transmit power is given by $P_{\mathrm{s}, \ell}=P_{\mathrm{s}} / L$, influencing the half-level transmit amplitude $U_{\mathrm{s}, \ell}$ for each MIMO.

\subsection{SVD-based Resource Allocation}

Considering the SVD layer model, the noise power is unchanged at the receiver. However, the half vertical eye openings $U_{\mathrm{A}}$ at each time slot $k$ and layer $\ell$ are influenced by the singular values so that $U_{\mathrm{A}}^{(\ell, k)}=\sqrt{\xi_{\ell, k}} U_{\mathrm{s}, \ell}$ holds and the corresponding SNR values are given by

$$
\rho_{\mathrm{SVD}}^{(\ell, k)}=\frac{\xi_{\ell, k} U_{\mathrm{s}, \ell}^{2}}{P_{\mathrm{R}}}=\frac{3 \xi_{\ell, k}}{L\left(M_{\ell}-1\right)} \frac{E_{\mathrm{s}}}{N_{0}},
$$

with $E_{\mathrm{s}}$ being the transmit symbol energy and the parameter $N_{0}$ is describing the noise power spectral density. The overall bit-error rate of the uncoded MIMO system is largely determined by the layer with the highest BER. In order to balance the bit-error rates on all layers, the mean of choice is to equalize the SNR values $\rho_{\text {SVD }}^{(\ell, k)}$ over all layers. This is clearly not the optimal solution for minimizing the overall BER but it is easy to implement and not far away from the optimum as shown in [3]. Therefore, the half-level transmit amplitude $U_{\mathrm{s}, \ell}$ is adjusted on each layer by multiplying it with $\sqrt{p_{\ell, k}}$ in order to apply the power allocation (PA) scheme. Consequently, the half vertical eye opening of the received symbols becomes

$$
U_{\mathrm{A}, \mathrm{PA}}^{(\ell, k)}=\sqrt{p_{\ell, k}} \sqrt{\xi_{\ell, k}} U_{\mathrm{s}, \ell} .
$$

With this adjustment the SNR values result in

$$
\rho_{\mathrm{PA}}^{(\ell, k)}=p_{\ell, k} \rho_{\mathrm{SVD}}^{(\ell, k)} \text {. }
$$

The overall transmit power after PA needs to be the same as without PA and thus

$$
P_{\mathrm{s}}=\sum_{\ell=1}^{L} p_{\ell, k} \cdot P_{\mathrm{s}, \ell}=\frac{P_{\mathrm{s}}}{L} \sum_{\ell=1}^{L} p_{\ell, k} \quad \forall k,
$$

has to be guaranteed. By combining these two requirements the PA factors $p_{\ell, k}$ for SVD-based systems can be calculated as follows [3]

$$
p_{\ell, k}^{(\mathrm{SVD})}=\frac{\left(M_{\ell}-1\right)}{\xi_{\ell, k}} \frac{L}{\sum_{\lambda=1}^{L} \frac{\left(M_{\lambda}-1\right)}{\xi_{\lambda, k}}} .
$$

Using the equal-SNR criterion nearly the same BER can be obtained on all activated layers. The only difference between 
the optimum PA and the proposed equal SNR PA is the consideration of the factor $2 /\left(\log _{2} M_{\ell}\right) \cdot\left(1-1 / \sqrt{M_{\ell}}\right)$ by the optimum PA. However, their influence, introduced by the layer-specific QAM constellation sizes, is far too small to generate remarkable differences in the performance [3].

In the SVD-based model the singular values are varying for each time slot. Therefore, an additional optimization is applicable by not only equalizing the SNRs for all layers at a given time slot but also by averaging over the number of time-slots per block. Considering the total available transmit power, the condition

$$
K \cdot L=\sum_{\ell=1}^{L} \sum_{k=1}^{K} p_{\ell, k}
$$

needs to be respected. As a result, the power allocation factors for layer and time-based PA in SVD systems can be calculated as follows

$$
p_{\ell, k}^{(\mathrm{SVD})}=\frac{\left(M_{\ell}-1\right)}{\xi_{\ell, k}} \frac{K \cdot L}{\sum_{\lambda=1}^{L} \sum_{\kappa=1}^{K} \frac{\left(M_{\lambda}-1\right)}{\xi_{\lambda, \kappa}}}
$$

and guarantee the above mentioned equal-SNR scenario for all activated layers and time slot per transmitted block. An illustration of the resulting SNRs of the proposed PA schemes for SVD systems is depicted in Fig. 4.

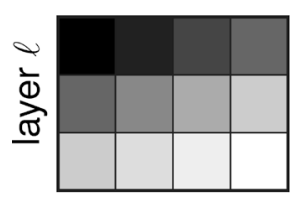

time $k$

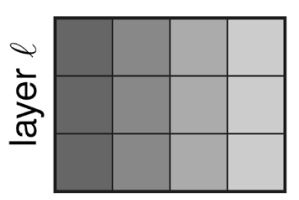

time $k$

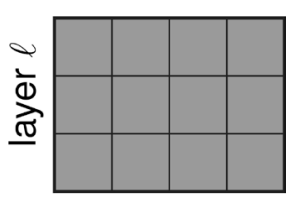

time $k$
Figure 4. Illustration of the remaining SNRs in SVD systems without applying PA (left), with layer-based PA (center) and with combined layer and time PA (right). The color black refers to high and white to low SNR values

As mentioned before, by using SVD-based block transmission the achievable SNR depends on the layer specific weighting factors as well as the chosen QAM constellation size. Whereas the layer-specific PA tries to balance the SNR at a given time-slot, the combined layer and time-based PA approach guaranties the same SNR on all activated layers and time-slots per transmitted block.

\subsection{T-PMSVD-based Resource Allocation}

By applying T-PMSVD the ISI is fully removed by the equalizer and thus for each layer the half vertical eye opening $U_{\mathrm{A}, \ell}$ of the receive signal equals the half-level amplitude of the transmitted symbol $U_{\mathrm{s}, \ell}$. The drawback of the T-PMSVD is that the noise and hence the noise power is weighted differently on each layer by the equalizer coefficients expressed by the factor $\theta_{\ell}$ so that the noise power on each layer results in

$$
P_{\mathrm{R}, \ell}=\theta_{\ell} P_{\mathrm{R}}, \quad \text { where } \theta_{\ell}=\sum_{\forall k}\left|f_{\ell, k}\right|^{2} .
$$

Therefore, the SNR values are resulting in

$$
\rho_{\mathrm{T}-\mathrm{PMSVD}}^{(\ell)}=\frac{U_{\mathrm{s}, \ell}^{2}}{\theta_{\ell} P_{\mathrm{R}}}=\frac{3}{\theta_{\ell} L\left(M_{\ell}-1\right)} \frac{E_{\mathrm{s}}}{N_{0}} .
$$

Equalizing the SNRs on all layers is achieved by varying the half-level transmit amplitude with the PA factor, where the factor is calculated as follows

$$
p_{\ell}^{(\mathrm{T}-\mathrm{PMSVD})}=\theta_{\ell}\left(M_{\ell}-1\right) \frac{L}{\sum_{\lambda=1}^{L} \theta_{\lambda}\left(M_{\lambda}-1\right)}
$$

and equals the beforehand mentioned combined layer and time PA SVD-based equal-SNR approach. The resulting SNRs for the proposed PA scheme in T-PMSVD systems are visualized in Fig. 5.

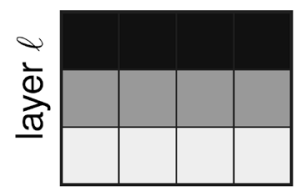

time $k$

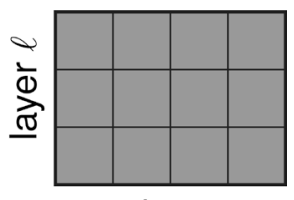

time $k$
Figure 5. Illustration of the remaining SNRs in T-PMSVD systems without applying PA (left) and with layer-based PA (right). The color black refers to high and white to low SNR values

\section{Results}

In this work, the BER quality is studied by using fixed transmission modes with a spectral efficiency of $8 \mathrm{bit} / \mathrm{s} / \mathrm{Hz}$. The analyzed QAM constellations, equivalent to how many bits are allocated to each layer, are shown in Table 1.

\begin{tabular}{|c|c|c|}
\hline throughput & layer 1 & layer 2 \\
\hline $8 \mathrm{bit} / \mathrm{s} / \mathrm{Hz}$ & 256 & 0 \\
\hline $8 \mathrm{bit} / \mathrm{s} / \mathrm{Hz}$ & 64 & 4 \\
\hline $8 \mathrm{bit} / \mathrm{s} / \mathrm{Hz}$ & 16 & 16 \\
\hline
\end{tabular}

Table 1: Transmission constellations

To analyze the T-PMSVD, a time-invariant $(2 \times 2)$ MIMO system is investigated. The polynomial channel matrix is chosen as

$$
\underline{\boldsymbol{H}}(z)=\boldsymbol{H}_{0}+\boldsymbol{H}_{1} z^{-1} \text { with } \boldsymbol{H}_{0}=\frac{4}{5}\left[\begin{array}{cc}
1 & 0.6 \\
0.5 & 0.8
\end{array}\right]
$$

and $\boldsymbol{H}_{1}=\boldsymbol{H}_{0} / 2$. The factor $4 / 5$ is taken to guarantee that the channel is not amplifying in any power allocation situation. The calculated BER results as a function of the symbol energy to the noise power spectral density $E_{\mathrm{s}} / N_{0}$ are depicted in Figure 6 and 7. 


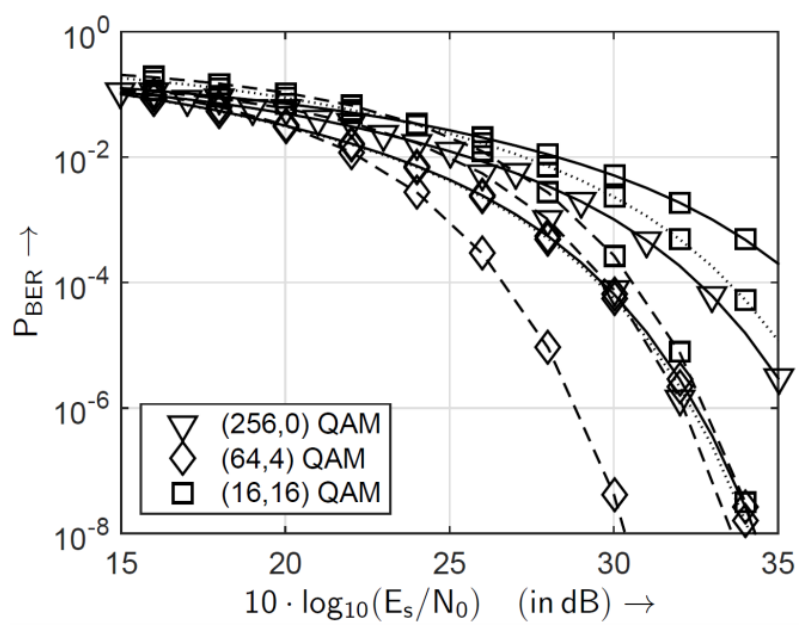

Figure 6. BER with layer and time-based PA (dashed line), with layer-specific PA (dotted line) and without PA (solid line) applying SVD-based equalization when transmitting over the time-invariant $(2 \times 2)$ MIMO channel

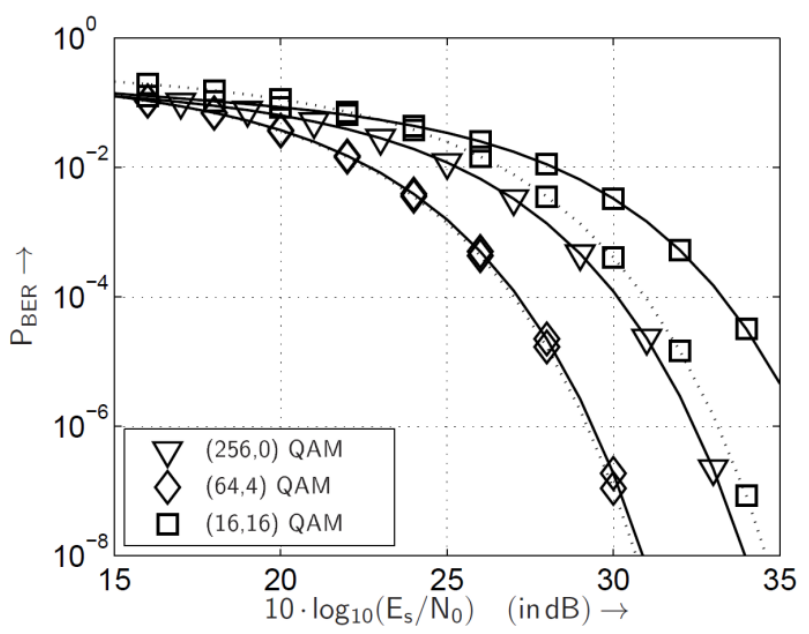

Figure 7. BER with PA (dotted line) and without PA (solid line) applying T-PMSVD equalization when transmitting over the time-invariant $(2 \times 2)$ MIMO channel

Here the $(64,4)$ QAM transmission mode shows the best results. The benefits of using the layer-based equal SNR PA are visible. For SVD systems further improvements in the resulting BER can be obtained by applying combined layer and time-based PA.

A direct comparison of the different transmission schemes is shown in Fig. 8, where the SVD based equalization with layer and time dependent PA performs slightly better than TPMSVD results with layer-based power allocation. However, the performance of T-PMSVD can be significantly increased by using an optimal detector such as a maximum-likelihoodsequence estimator, entitled as V-PMSVD. Here the available transmit power is equally split between the activated layers and the question of an optimized PA scheme remains as an open point for further studies.

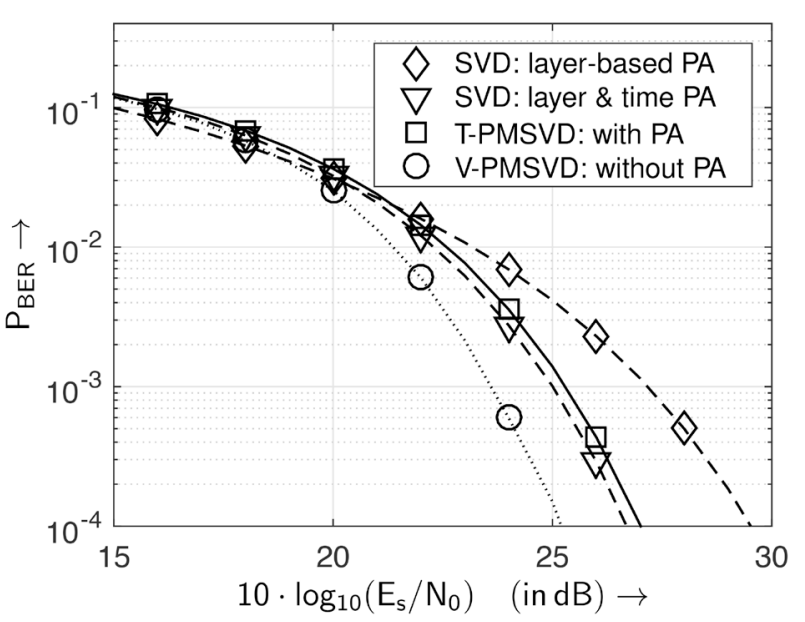

Figure 8. BER comparing different MIMO equalization techniques combined with different power allocation strategies transmitting with the $(64,4)$ QAM scheme over the time-invariant $(2 \times 2)$ MIMO channel

Spatial multiplexing in the optical domain can be realized by transferring multiple modulated light signals over different optical mode groups through a single multi-mode fiber (MMF) [1,7]. The excitation of different optical mode groups can be carried out by varying the light launch eccentricity. However, launching two different light sources into a single MMF with the desired eccentricities is practically complex at the present time. In this work fusion couplers are used for mode combining and splitting [7]. In order to apply MIMO the specific impulse responses need to be estimated. Here, the measurement results within a $1.4 \mathrm{~km}(2 \times 2)$ optical MIMO channel at an operating wavelength of $1576 \mathrm{~nm}$ have been used [6].

The corresponding BER results are depicted in Figure 9 and 10 for the different QAM constellation sizes. The $(256,0)$ transmission scheme shows the best results.

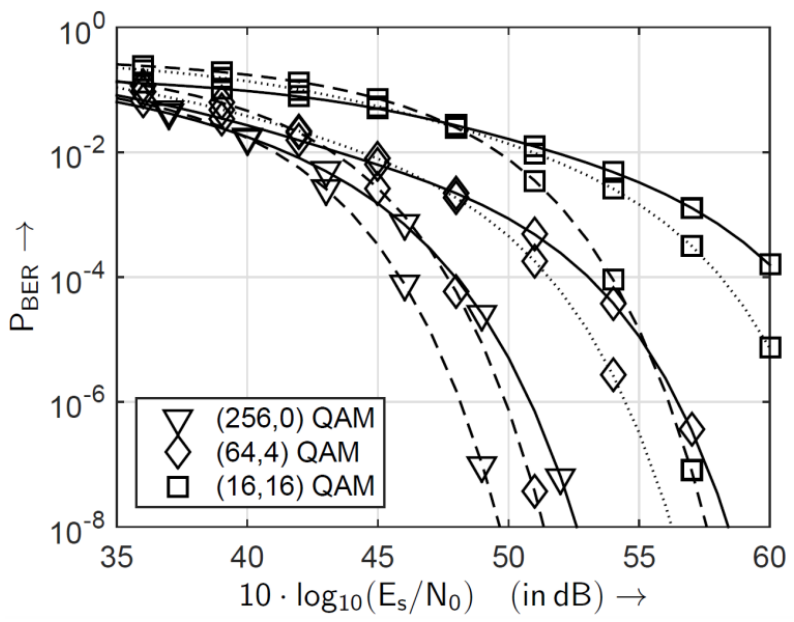

Figure 9. BER with layer and time-based PA (dashed line), with layer-specific PA (dotted line) and without PA (solid line) applying SVD-based equalization when transmitting over the optical $(2 \times 2)$ MIMO channel 


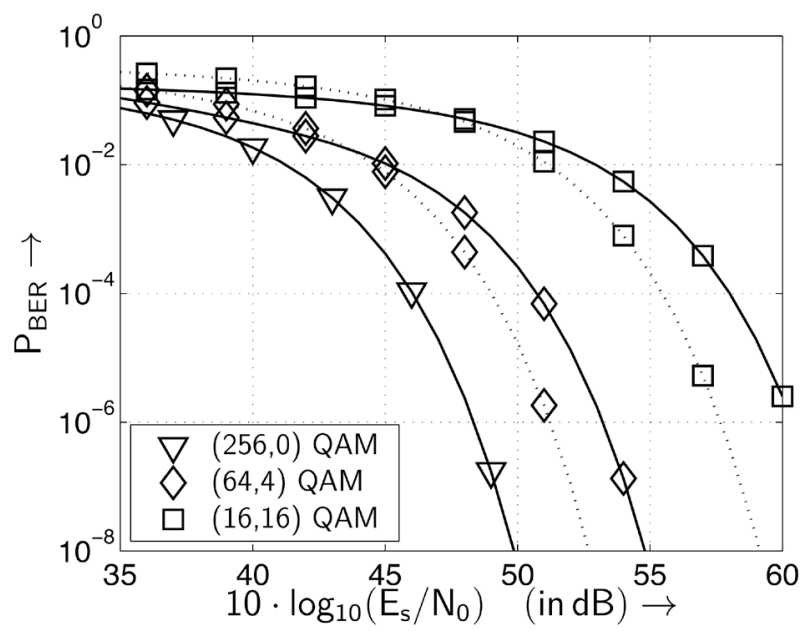

Figure 10. BER with PA (dotted line) and without PA (solid line) applying T-PMSVD equalization when transmitting over the measured $1.4 \mathrm{~km}$ optical $(2 \times 2)$ MIMO channel at an operating wavelength of $1576 \mathrm{~nm}$

Comparing the power allocation schemes shows that PA is again helpful when minimizing the overall BER. Moreover, by applying the combined layer and time-based PA, the BER performance increases significantly.

A direct comparison depicted in Figure 11 shows that SVD with combined layer and time-based PA performs similar to T-PMSVD equalization with PA. Transmitting with a bandwidth efficiency of $8 \mathrm{bit} / \mathrm{s} / \mathrm{Hz}$ and considering the used symbol-frequency of $f_{\mathrm{T}}=1 / T_{\mathrm{s}}=620.347 \mathrm{MHz}$, a bit-rate of approximately $5 \mathrm{Gbps}$ is obtained.

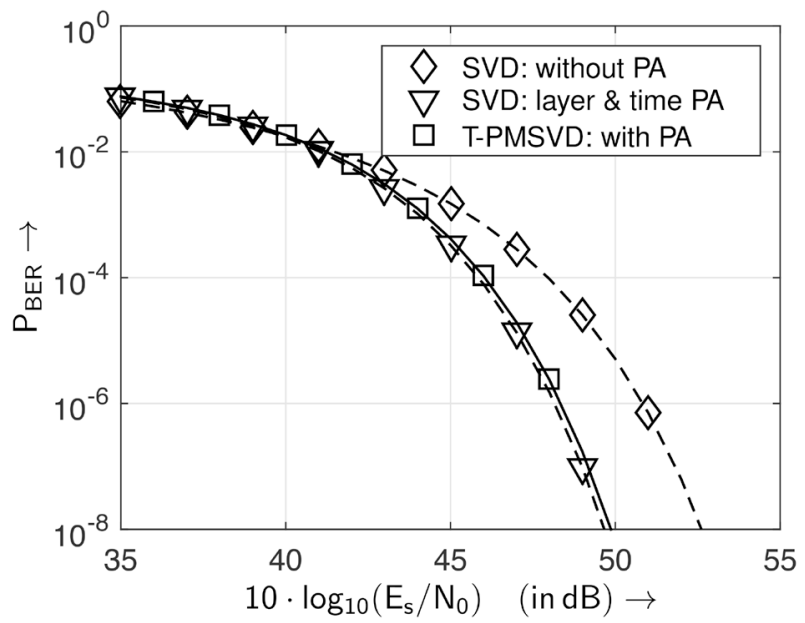

Figure 11. BER comparing different MIMO equalization techniques combined with different power allocation strategies transmitting with the $(256,0)$ QAM scheme over the optical $(2 \times 2)$ MIMO channel

\section{Conclusions}

In this paper broadband MIMO systems have been described using polynomial matrix factorization. In order to remove the MIMO channel interference a singular value decomposition algorithm for polynomial matrices (PMSVD) including layerspecific $\mathrm{T}$-spaced equalization for eliminating the remaining inter symbol interference has been studied. This T-PMSVD technique has been compared in terms of the bit-error rate performance with the well-known spatio-temporal vector coding description applying SVD equalization. For both equalization types bit loading schemes have been combined with equal SNR power allocation in order to optimize the BER performance. The equal SNR criterion for power allocation seems to be a good sub-optimum solution for improving the channel performance. Furthermore, the bit and power loading analogies between both equalization types have been shown. The investigated channels show that the optimal QAM transmission mode largely depends upon the used channel type and that the activation of all transmission layers does not necessarily lead to the best performance. Here, PMSVD systems show to be an alternative to SVDbased systems.

\section{Acknowledgement}

The authors thank Professor John McWhirter from Cardiff University (UK) for many valuable comments and fruitful discussions on the topic investigated in this contribution.

\section{References}

[1] P. J. Winzer, G. J. Foschini. "MIMO Capacities and Outage Probabilities in Spatially Multiplexed Optical Transport Systems", Optics Express, 17(19) 2011

[2] A. C. Singer, N. R. Shanbhag, H.-M. Bae. "Electronic Dispersion Compensation - An Overview of Optical Communications Systems", IEEE Signal Processing Magazine, 25(6) 2008, pp. $110-130$.

[3] A. Ahrens, C. Benavente-Peces. "Modulation-Mode and Power Assignment in Broadband MIMO Systems", Facta Universitatis (Series Electronics and Energetics) 22.3 (2009), pp. 313-327.

[4] J. McWhirter, P. Baxter, T. Cooper, S. Redif, J. Foster. "An EVD Algorithm for Para-Hermitian Polynomial Matrices”, IEEE Transactions on Signal Processing, vol. 55, no. 5, May 2007, pp. 2158-2169.

[5] G. G. Raleigh, J. M Cioffi. "Spatio-Temporal Coding for Wireless Communication", IEEE Transactions on Communications 46.3 (1998), pp. 357-366.

[6] A. Sandmann, A. Ahrens, S. Lochmann. "Resource Allocation in SVD-Assisted Optical MIMO Systems using Polynomial Matrix Factorization", ITGFachbericht 257: Photonische Netze. Leipzig (Germany), 2015, pp. 128-134.

[7] A. Sandmann, A. Ahrens, S. Lochmann. "Experimental Description of Multimode MIMO Channels utilizing Optical Couplers", ITG-Fachbericht 248: Photonische Netze. Leipzig (Germany), 2014, pp. 125-130. 